DOI: https://doi.org/10.11144/Javeriana.umed59-2.hemi

\title{
Hemimelia del peroné y la tibia, más que un hueso ausente: presentación de casos
}

\section{Fibular and Tibial Hemimelia, More than Just an Absent Bone: Case} Presentations

Fecha de recepción: 18/07/2017 | Fecha de aprobación: 25/07/2017

\author{
María Camila Medina ${ }^{\mathrm{a}}$ \\ Pontificia Universidad Javeriana, Colombia \\ Juana María Vallejo \\ Pontificia Universidad Javeriana, Colombia \\ Alejandra Torres Mejía \\ Pontificia Universidad Javeriana, Colombia \\ Javier Cogollo \\ Pontificia Universidad Javeriana, Colombia
}

a Correspondencia: medina.camila01@gmail.com

Cómo citar: Medina MC, Vallejo JM, Torres Mejía A, Cogollo J. Hemimelia del peroné y la tibia, más que un hueso ausente: presentación de casos. Univ. Med. 2018;59(2). doi: https://doi.org/10.11144/ Javeriana.umed59-2.hemi

\section{RESUMEN}

El acortamiento congénito del miembro inferior hace parte de un grupo de trastornos poco frecuentes, entre los que se encuentran la deficiencia femoral focal proximal y la hemimelia de peroné o de la tibia. Pueden presentarse desde una forma leve de hipoplasia hasta ausencia completa del hueso con grados variables de acortamiento, siendo evidente el acortamiento de la extremidad desde el nacimiento. El artículo revisa este tema y presenta los casos de hemimelia de tibia y peroné del Hospital Universitario San Ignacio en los últimos años, se describen los principales hallazgos imaginológicos en radiografía simple y en resonancia magnética. Palabras clave

anomalía del desarrollo; hueso/esqueleto; pediatría; peroné; hemimelia del peroné; tibia; hemimelia de la tibia.

\begin{abstract}
Congenital lower extremity shortening is part of a group of infrequent disorders, together with proximal femoral deficiency, tibial and fibular hemimelia. It can manifest as a mild form of hypoplasia to a complete lack of the bone, with variable degrees of shortening and with an evident start since birth. In this pictorial review, we will describe cases of fibular and tibial hemimelia seen at San Ignacio University Hospital in the last years. The main imaging findings are described in X ray and MRI.

Keywords

bone/skeleton; developmental anomaly; pediatric; fibula; fibular hemimelia; tibia; tibial hemimelia; imaging.
\end{abstract}

\section{Introducción}

La hemimelia del peroné es el acortamiento congénito de los huesos largos más frecuente, seguida por la agenesia o hipoplasia de la tibia, el cúbito, el radio y el fémur, respectivamente (1). Tiene una incidencia de 7,4 a 20 casos 
por cada millón de recién nacidos vivos y es dos veces más frecuente en hombres que en mujeres (2). En la mayoría de los casos se presenta de manera aislada y esporádica. Sin embargo, puede hacer parte de un síndrome con múltiples anomalías asociadas (1). La hemimelia abarca un espectro de anomalías, desde la más leve, con ligero acortamiento del hueso, a la más grave, con ausencia total.

La hemimelia de la tibia es una anormalidad mucho más rara que la del peroné. Tiene una incidencia aproximada de una en un millón, y a diferencia de la hemimelia del peroné, que es esporádica, esta tiene un patrón de herencia autosómico dominante $(2,3)$.

El diagnóstico precoz y la clasificación radiológica son imprescindibles para el manejo y la planeación quirúrgica, siendo la radiografía y la resonancia magnética las principales modalidades diagnósticas para su evaluación.

\section{Presentación de casos}

Los siguientes casos corresponden a pacientes menores de edad, quienes se encontraban en estudio prequirúrgico y genético de la hemimelia del peroné o la tibia en el Hospital Universitario San Ignacio en los últimos 4 años.

El primer caso documentado es de un bebé de 6 meses de edad, a quien en estudios prenatales se le había detectado ausencia del peroné derecho y oligodactilia por agenesia del quinto metacarpiano (figura 1). En el examen físico presentaba acortamiento del miembro inferior izquierdo con arqueamiento anterior de la pierna, pie equino valgo, oligodactilia e inestabilidad de la rodilla por hipoplasia del ligamento cruzado anterior (figura 2).
Figura 1.Radiografía de miembros inferiores y pies
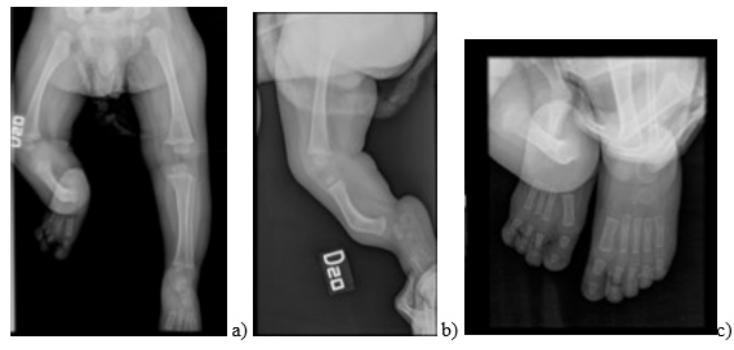

a y b) Ausencia completa del peroné derecho, así como arqueamiento anterior de la tibia y disminución en la longitud de la extremidad inferior derecha a expensas del componente tibial. c) Ausencia de los núcleos de osificación del hueso cuboides, primera cuña del pie derecho y quinto dedo

Figura 2.Resonancia magnética (DP)
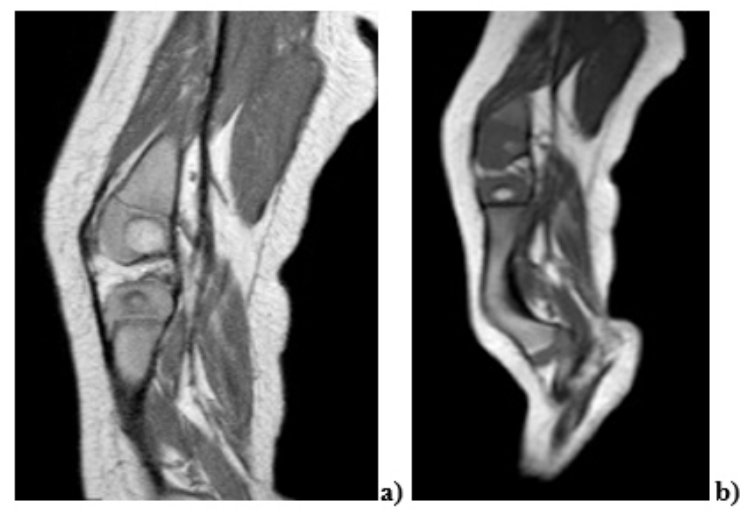

a) Hipoplasia del ligamento cruzado anterior de la rodilla derecha. b) Hay acortamiento y arqueamiento anterior de la tibia derecha con marcada atrofia muscular

El segundo caso corresponde a un paciente de 11 años de edad con inestabilidad de la rodilla y acortamiento del miembro inferior izquierdo en estudios prequirúrgicos de la hemimelia de peroné. Se le realizó una resonancia magnética de rodilla con signos de inestabilidad de la articulación de la rodilla, por ausencia completa del ligamento cruzado anterior (figura 3), así como inestabilidad de la patela. 
Figura 3.Resonancia magnética (DP)

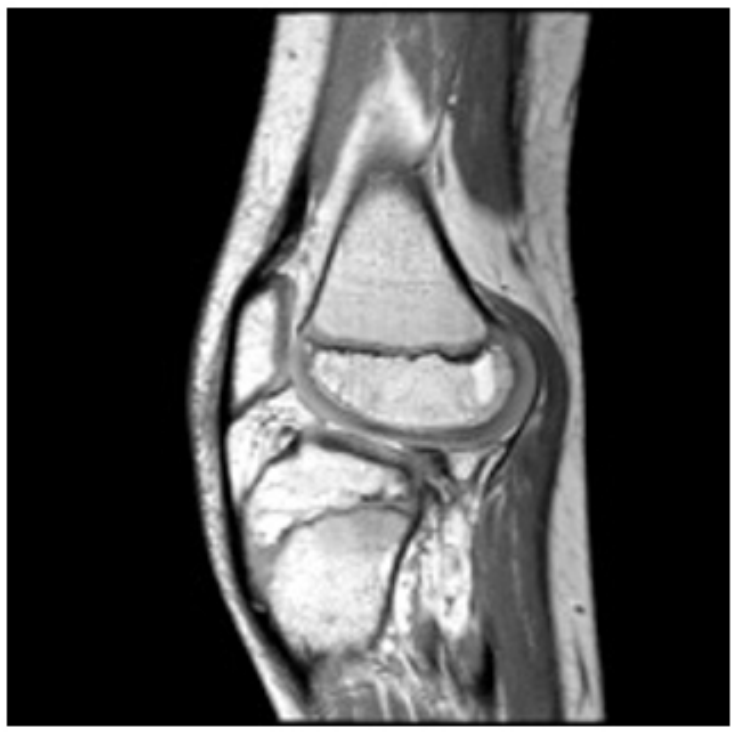

Se observa agenesia del ligamento cruzado anterior con translación anterior de la tibia, patela baja e inserción baja del tendón patelar

El tercer caso fue evidenciado en un paciente de 4 años de edad con hemimelia de la tibia, dado por hipoplasia bilateral de la tibia. Asociado, presentaba polidactilia, un compartimento posterior deficiente con pie equino-varo (figura 4).

Figura 4.Resonancia magnética en T2

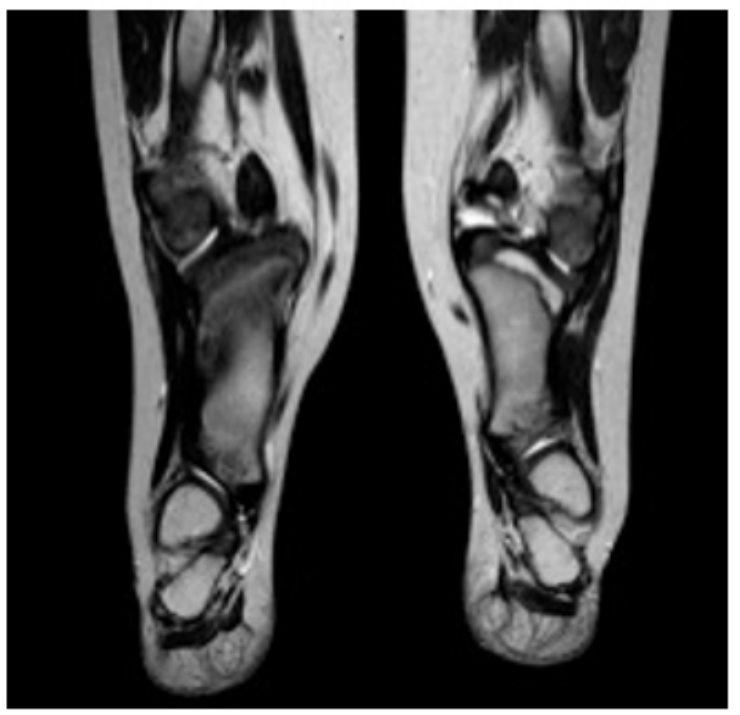

Compartimento anterior deficiente y ausencia de ligamentos colaterales

\section{Discusión}

La hemimelia del peroné puede tener afectación uni o bilateral, siendo más frecuente la unilateral, principalmente del lado derecho, en el 80\% de los casos. Se han descrito diferentes teorías que explican esta condición, entre las cuales están la ausencia de la arteria tibial anterior y pedia, bandas amnióticas y defectos del ectodermo en el primer trimestre del embarazo (4).

Generalmente, esta entidad se asocia con otras alteraciones del esqueleto, como la deficiencia femoral focal y la hipoplasia del cóndilo femoral lateral presente del 15\% al 70\% de los casos. Las anormalidades de la tibia pueden estar presentes o no como el acortamiento y el arquemiento anterior, deformidades del pie (pie equino valgo) y aparición tardía de los huesos del tarso, ausencia de falanges y metatarsianos usualmente del lado lateral del pie, así como malformaciones de la rodilla (inestabilidad, genu valgo o ausencia del ligamento cruzado anterior).

Hay una fuerte asociación entre el acortamiento del peroné y las anormalidades del pie; entre más severo el acortamiento, mayor agenesia de los metatarsianos. La coalición tarsiana reduce la movilidad del retropié, siendo el patrón más frecuente la unión talocalcanea fibrosa.

Existen diferentes clasificaciones de la hemimelia fibular congénita. La clasificación radiológica más utilizada es la de Achterman y Kalamchi (5). Esta describe los hallazgos radiológicos del peroné sin considerar los hallazgos asociados del tobillo o el pie (figura 5). 
Figura 5.Clasificación de Achterman y Kalamachi para hemimelia fibular
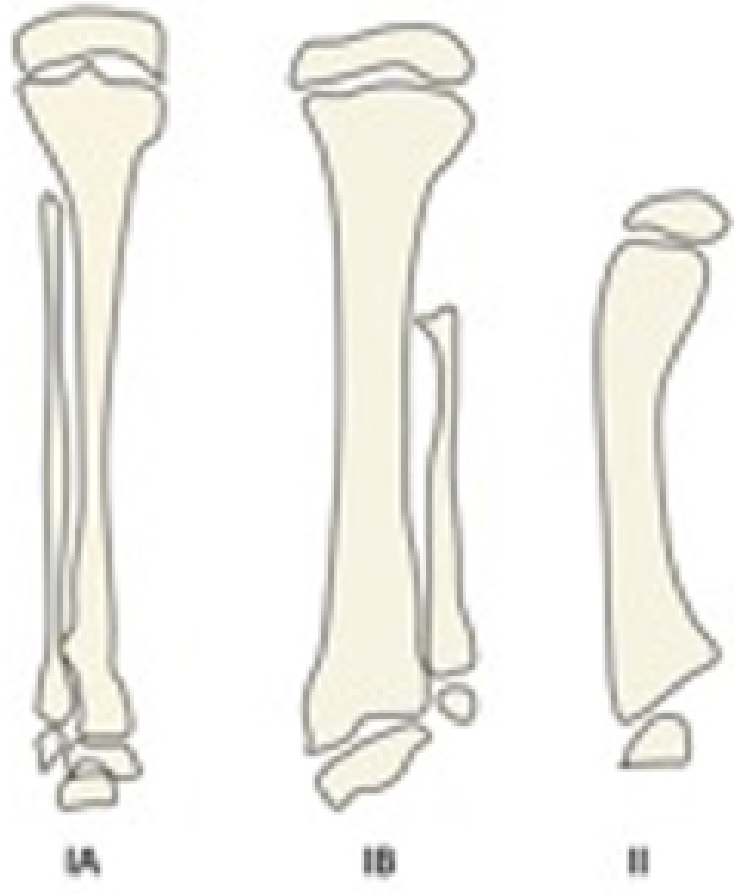

Tipo I (hipoplasia), IA: la epífisis proximal del peroné se encuentra más distal a la placa de crecimiento tibial; mientras que la placa de crecimiento del peroné distal es proximal a la cúpula del astrágalo. IB: hay ausencia parcial de peroné proximal del 30\% al 50\%; en tanto que en su tercio distal está presente, pero no puede soportar el tobillo.

Tipo II (aplasia): ausencia completa del peroné o cuando existe un pequeño fragmento distal del peroné (1).

En la hemimelia de la tibia, al igual que en la hemimelia del peroné, el hueso deficiente es usualmente remplazado por una banda fibrosa o fibrocartilagenosa. En neonatos e infantes, la diferencia entre estas dos puede ser difícil, porque el peroné puede estar hipertrofiado; sin embargo, el peroné no está arqueado y no se articula con el fémur distal. El peroné puede estar normal, displásico o en una posición alta o subluxado y con la desviación del pie hacia adentro y no hacia fuera, como la hemimelia del peroné. Las anomalías anatómicas más frecuentes asociadas son: una suplencia vascular anormal del miembro inferior, displasia o ausencia de músculos (en especial del compartimento posterior profundo y anterior), deficiencia congénita del fémur, fémur bífido, ausencia de la patela, una articulación peroneoastragalina-calcánea (en vez de una verdadera articulación del tobillo) y polidactilia (5).

En la clínica predomina la inestabilidad multidireccional de la rodilla y el tobillo (por la ausencia de una articulación normal), deformidad en equinovaro, mecanismo extensor deficiente y una discrepancia en la longitud del miembro inferior que alcana de 18 a $20 \mathrm{~cm}$ con la madurez esquelética (6).

La clasificación de Jones ha dispuesto la hemimelia de la tibia en cuatro tipos, según los hallazgos radiológicos (figura 6): 1) tipo IA, en que hay ausencia completa de la tibia y el núcleo de osificación del fémur distal es pequeño o ausente; 2 ) tipo $\mathrm{Ib}$, en que la porción cartilaginosa de la tibia está presente en nacimiento, pero no está osificada (puede verse por ecografía o resonancia magnética). 3) tipo II, en que la porción proximal de la tibia está presente en el nacimiento, pero la porción distal no se ve. Y 4) tipo IV, en que la tibia es corta y hay una diástasis tiobioperonea, la superficie articular de la tibia distal está ausente y hay desplazamiento proximal del astrágalo (5).

Figura 6.Clasificación de Jones para la hemimelia de la tibia
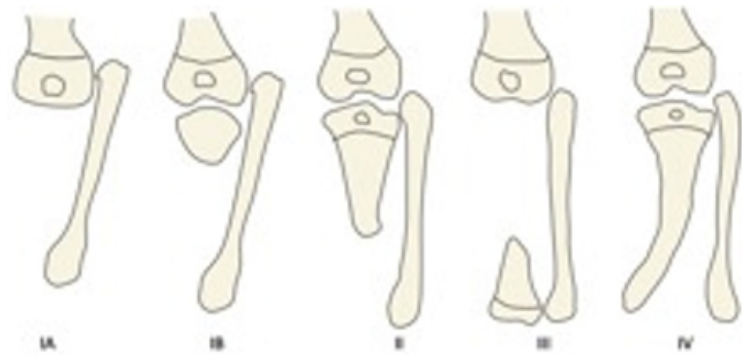

\section{Evaluación radiológica}

En condiciones normales, el margen superior de la epífisis proximal del peroné debe estar a nivel de la fisis, proximal de la tibia, y la fisis distal del peroné debe estar a nivel del domo talar.

Es mandatoria la evaluación radiológica completa de la cadera y del pie, por las múltiples malformaciones asociadas. La 
radiografía puede sobrestimar o subestimar la extensión de las malformaciones. Por ejemplo, en pacientes jóvenes, la coalición tarsiana de patrón cartilaginoso no puede ser reconocida en la radiografía.

El objetivo de la radiografía es caracterizar el grado de hipoplasia o agenesia. Así mismo, evaluar el acortamiento tibial, femoral o del peroné; el compromiso epifisiario, la deformidad en valgo o el malalineamiento, que puede estar representado por esclerosis cortical y fracturas por estrés.

La resonancia magnética ayuda a detectar las deformidades asociadas de los tejidos blandos, la estabilidad articular y las bandas fibrosas o fibrocartilaginosas.

\section{Conclusiones}

El acortamiento congénito de las extremidades inferiores son patologías complejas con una variedad de anomalías anatómicas. El rol de radiólogo está en el diagnóstico, la evaluación prequirúrgica y el seguimiento; por esto la importancia de conocer el espectro de estas anomalías para un tratamiento temprano y preciso.

\section{Referencias}

1. Bedoya MA, Chauvin NA, Jaramillo D, Davidson R, Horn D, HoFung V. Common patterns of congenital lower extremity shortening: Diagnosis, classification, and follow-up. RadioGraphics. 2015;35(4):1191-1207.

2. Renjifo Correa E, Mantilla Espinosa R, Pareja Sierra AM, Monsalve Villamizar J. Hemimelia fibular: presentación de un caso. Rev Colomb Radiol. 2014;25(1):3896-98.

3. Yoong P, Mansour R. Internal derangement of the knee in fibular Hemimelia. The Knee. 2014 Jun;21(3):749-56.

4. Kim JK, Baek GH, Chung MS, Lee SK. Terminal Hemimelia of the lower extremity: absent lateral ray and a normal fibula. Int Orthop. 2008;32:263-7.

5. Paley D, Chong DY. Tibial hemimelia. En: Sabharwal S, editor. Pediatric lower limb deformities. Springer International Publishing Switzerland; 2016.

6. Laor T, Jaramillo D. MR imaging in congenital lower limb deformities. Pediatr Radiol. 1996;26:381-87. 